\title{
EDITORIAL
}

\section{Sidecountry Rescue-Who Should Respond to Ski Resort Out-of-Bounds Rescues?}

The latest buzzword in winter recreation, "sidecountry skiing and snowboarding," is popping up in magazines, equipment marketing materials, and ski resort websites. The increasing popularity poses several questions. ${ }^{1,2}$ First, what or where is the "sidecountry" aka "slackcountry?" Is it a geographic local, an activity, or both? Simply defined, the sidecountry is the area adjacent to but outside the boundary of winter resorts, which is accessed from the resort by a paying customer. In other words, a skier or snowboarder buys a lift ticket, rides a lift, and then heads out of bounds. This raises many questions and concerns. The biggest quandary: who should respond to sidecountry rescues?

This author presented this topic for discussion at conferences of 3 distinct groups: the National Ski Patrol Farwest Division Winter Conference, the Mountain Rescue Association 50th Anniversary Conference, and the 2009 Wilderness Medical Society Annual Meeting. Discussions centered on liability for rescuers, timely response, and the feasibility of joint trainings.

\section{Why Head Into the Sidecountry?}

Every year, thousands of accidents occur to skiers and snowboarders within the boundaries of the winter sports resorts or ski areas. Known as "in area" skiing and snowboarding, safety is monitored and enhanced by professional and volunteer ski patrollers, who operate as employees of the winter resort company. Accidents also occur in the backcountry, the area outside the boundaries or operating hours of developed resorts. Of 50000 search and rescue missions per annum, 1300 occur in Colorado alone, with 6\% of those relating to skiers/snowboarders. ${ }^{3}$ In most states and Canadian providences, law enforcement agencies are responsible for backcountry rescues and use volunteer mountain search and rescue teams. Adjunct participants may include personnel from private ambulance companies, city or county fire rescue teams, military units, the forest service, or ski patrols.

Leaving the ski resort into the sidecountry, which is technically part of the backcountry, can be legal. If allowed, resorts yield access through a specific break in the resort perimeter, called a "control gate," which may or may not be opened by the ski patrol on any given day depending on safety conditions of weather, terrain, and snowpack. Boundary lines that are intermittently open are called "soft boundaries." However, it is unlawful in most states for a skier or snowboarder to exit the ski resort when the boundary is closed. Some resorts keep their boundary closed permanently—referred to as "hard boundaries."

Sidecountry skiing and snowboarding provides a backcountry experience with much less physical effort and time than hiking directly from a parking lot or trailhead. Compared to resort skiing, the sidecountry, like the backcountry, allows access to different terrain and snow conditions, often untracked powder, for example.

Unfortunately, the sidecountry can create a false sense of security with easy access, close proximity to the resort, and escalating popularity. The sidecountry often allows skiers and snowboarders with limited or no backcountry experience to venture beyond the relative safety of ski resort boundaries. Within ski areas, obstacles are marked, runs are groomed, ski patrollers come to the aid of customers, and certain terrain and snow features are made safe, such as avalanche zones and cliffs. The sidecountry, like the backcountry, is replete with a full cadre of mountain hazards, namely, avalanches, unmarked obstacles, cliffs, and routes of variable conditions like ice and deep snow.

In a related matter, "uphill traffic" within ski resorts has also increased in popularity. Alpine touring skiers, telemark skiers, and snowboarders ascend ski runs using climbing skins or snowshoes, without getting on a lift. Uphill traffic participants get exercise, learn to use backcountry equipment, and have a sidecountry ski experience within the safety of the resort, often without buying a ticket. Ski resorts lie on either public land leased from the forest service or private land. In either case, they can disallow uphill traffic. With similar issues to sidecountry use, uphill traffic sometimes occurs in the morning or evening, when the ski resort is closed. 


\section{Who Comes to the Rescue?}

The biggest concern with sidecountry accidents is timely rescue. Search and rescue (SAR) teams are usually charged with rescues, dispatched under authority of county sheriffs or Royal Canadian Mounted Police. However, gearing up for a mission is time-consuming: volunteer rescuers leave work and family, rush home to assemble personal gear, and race up the mountain, often with little information. Although ski patrollers are in immediate proximity, they are not legally obligated to cover out of bounds and, due to liability issues, some ski areas have refused to let patrollers respond.

Don Schockey, Heavenly National Ski Patrol volunteer and coauthor of an International Snow Science Workshop paper Sidecountry Rescue: Who Is Responsible?, ${ }^{4}$ believes ski patrollers should head into the sidecountry. "We are paid to be in area, but with life-threatening, time-critical injuries in the sidecountry, we can get there the quickest with the right equipment. That gives the injured person the best chance of survival. Not only can ski patrollers provide immediate response, they are highly skilled and equipped, and they may know the sidecountry terrain best" (written communicaton, April 2009).

Paul Baugher, Crystal Mountain, Washington, Ski Patrol Director, agrees that patrollers are "specifically trained and equipped" and can respond fastest. "Winter rescues have a narrow time window before they become deadly serious," said Baugher (oral communication, April 12, 2009).

One problem with patrol response, said Schockey, is diminished resources in-area. "If the resort is short patrollers and equipment that could jeopardize the safety of paying customers."

A second concern: ski patrollers may have no liability and workers compensation insurance coverage outside the resort boundaries. In many states, the Good Samaritan Law is not applicable. In Oregon, for example, the Good Samaritan Law applies to physicians only. State emergency management insurance may not apply to ski patrollers either.

A third issue is that not all ski patrollers possess backcountry skills, terrain knowledge, fitness, and equipment such as alpine touring or telemark skis with climbing skins and an overnight backpack.

\section{Can We Solve the Problem?}

Both time-tested and innovative solutions are focused on rapid response to sidecountry incidents, while eliminating modernday problems of insurance and maintaining in-area staffing.

In Utah, full-time professional ski patrollers run the 22year-old Wasatch Backcountry Rescue (WBR). Dean Cardinale, President of WBR, said patrollers know the terrain, weather, and snowpack because they are in it daily. "We can take one team per resort so we don't deplete resorts of manpower," he explained. "We can have 9 teams from 9 resorts respond." Since WBR is dispatched by the sheriff's office, rescuers' liability and workers compensation insurances are covered, said Cardinale (oral communication, April 2009).

On Oregon's Mount Hood, the Portland Mountain Rescue (PMR) Ready Teams patrol in and out of bounds on busy weekends above Timberline Lodge Ski Area. Steve Rollins, PMR Rescue Leader, said, "Ready Teams are coordinated with Timberline Lodge. This allows for a rapid and competent response while allowing the ski patrol to carry on with their normal resort operations" (written communication, April 2009).

This author's mountain rescue team, Crag Rats in Hood River, OR, has members who work on the ski patrol and at the medical clinic at Mount Hood Meadows Ski Resort. Thus, we can respond as either an employee of the resort or hospital-run clinic, or as a member of the mountain rescue team.

A multi-agency approach using helicopters has been successful in Colorado and British Columbia. According to Charlie Shimanski, president of Mountain Rescue Association and 22-year veteran of Alpine Rescue Team, Denver's Flight for Life helicopter responds to backcountry and sidecountry rescues in Summit County, the "epicenter of avalanche fatalities" (oral communication, May 2009). The hospital-based helicopter can be dispatched under the authority of the sheriff, off load the flight nurse and paramedic, and ferry a team of ski patrollers or SAR volunteers to the scene in minutes. Both ski patrollers and SAR volunteers get training and credentials specifically for this program.

Similarly, Ian Foss, a rescue leader from Golden and District Search and Rescue in British Columbia, said that last year they responded to in-area and sidecountry rescues in less than 30 minutes using helicopters (oral communication, May 2009).

A few recommendations seem obvious, but somewhat difficult to employ. First, winter resorts should have a clear protocol with regards to sidecountry rescues so there is no confusion. Second, backcountry training, terrain knowledge, fitness, and equipment are as important for sidecountry-capable ski patrollers, as they are for volunteer SAR personnel. Third, if appropriate, ski patrols should coordinate sidecountry rescues with law enforcement agencies responsible for rescues. Finally, search and rescue teams and ski patrollers would benefit from joint trainings. However, joint trainings tend to be difficult. Teams in small communities in Colorado and Alaska reported joint training with ski patrols, fire rescue units, search dog teams, and mountain rescue teams. But coordinating multi-agency trainings is time-consuming and difficult. 
Even with improved response, complications like vague information, poor communication, and foul weather can delay rescues. Although obstacles of time, liability, and resource depletion can be minimized, that still does not guarantee rescue.

Christopher Van Tilburg, MD, FAWM

Mountain Medical Clinic

Providence Hood River Memorial Hospital

Hood River, OR

Medical Committee

Mountain Rescue Association

Golden, $\mathrm{CO}$

\section{References}

1. Stifler E. White peril: side country, backcountry skiing demands special caution. Lone Peak Lookout, February 7, 2008. www.lonepeaklookout.com. Accessed January 5, 2009.

2. Van Tilburg C. C. Sidecountry rescue. Backcountry. 2010; 15:24-25.

3. Finco L. Statistics for Calendar Year 2007. Golden, CO: Mountain Rescue Association; 2007:1-2.

4. Shockey D, Morrill C, Immeker D. Sidecountry rescuewho is responsible? Proceedings of the International Snow Science Workshop. Whistler, Canada. September 21-27, 2008:926-930. 